\title{
The
}

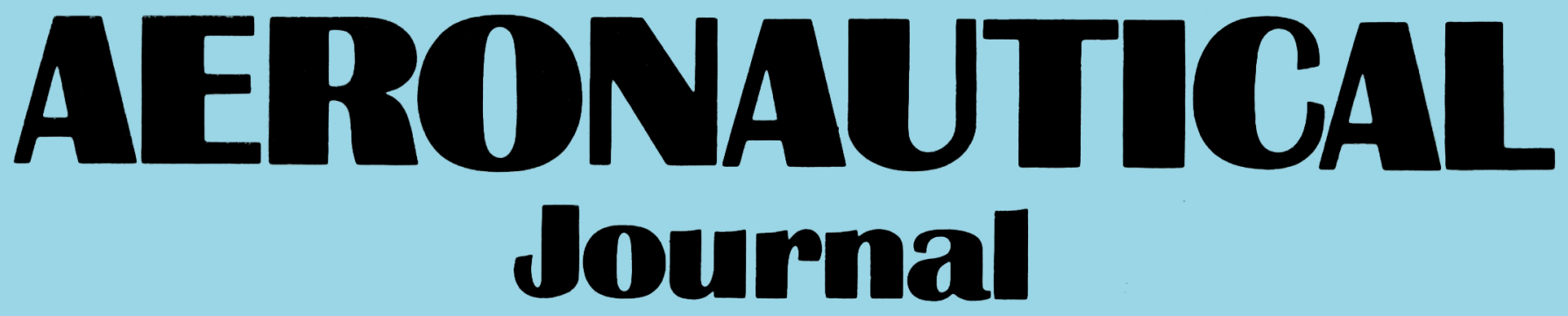

\section{EUROPEAN EDITION}

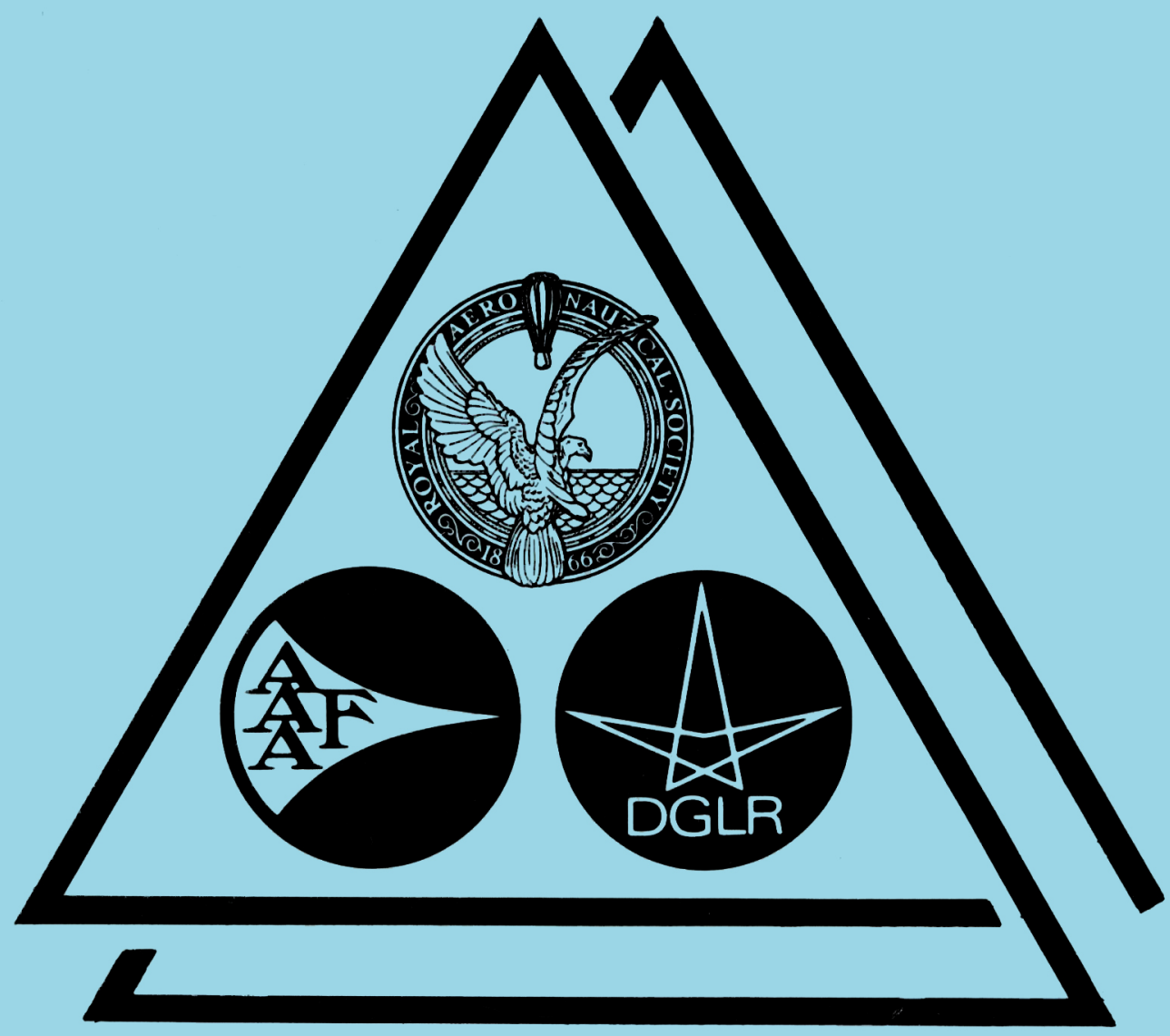

Incorporating

The Aeronautical Quarterly

Volume 92 Number 920 December 1988 


\section{NOTES ON THE PREPARATION OF PAPERS FOR PUBLICATION IN THE AERONAUTICAL JOURNAL}

All papers submitted for publication are sent to two referees.

Three clear copies of the manuscript, each complete with illustrations, should be submitted. Papers should be typed in double spacing and not exceed 10000 words. Handwritten manuscripts cannot be accepted.

A list of all symbols used, both in the illustrations and in the text, should be given in alphabetical order at the beginning of the paper whether or not they are standard. All unfamiliar terms must be explained.

Symbols and formulae should be typewritten if possible. The use of dots, bars and so on, over symbols, or the use of dots as multiplication signs and bars for brackets should be avoided. Suffixes and indices must be clearly indicated and complicated suffixes avoided.

References should be numbered in the text where they occur, and a complete list of references given at the end of the paper. (The Aeronautical Journal uses the BSI Numeric System for reference - eg, author(s), title of contribution, title of periodical, year of publication, volume number, page numbers.)

lilustrations should be kept to a minimum. When the paper is first submitted they can be clear prints or graphs, sketches or photographs. If the paper is accepted, authors are asked to provide the original drawings, if possible, and clear, glossy, black-and-white photographs. All diagrams should be produced to the same scale.

\section{Conditions of Publication}

An author must obtain consent, where necessary, to use any material in a paper which is copyright or the property of any other person or his employers.

Security clearance, if appropriate, must be obtained by the author before the paper is submitted.

The copyright of papers printed in the Aeronautical Journal is the property of The Royal Aeronautical Society. Permission to reprint or to use any paper will not be refused unreasonably.

Authors are entitled to 50 complimentary reprints of their papers. Authors may order any number of additional reprints at the time of printing and quotations can be provided. 

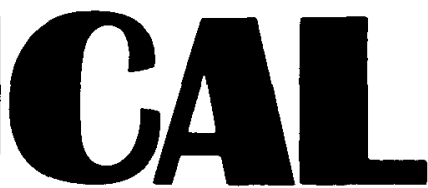

100 Incorporating the Aeronautical Quarterly

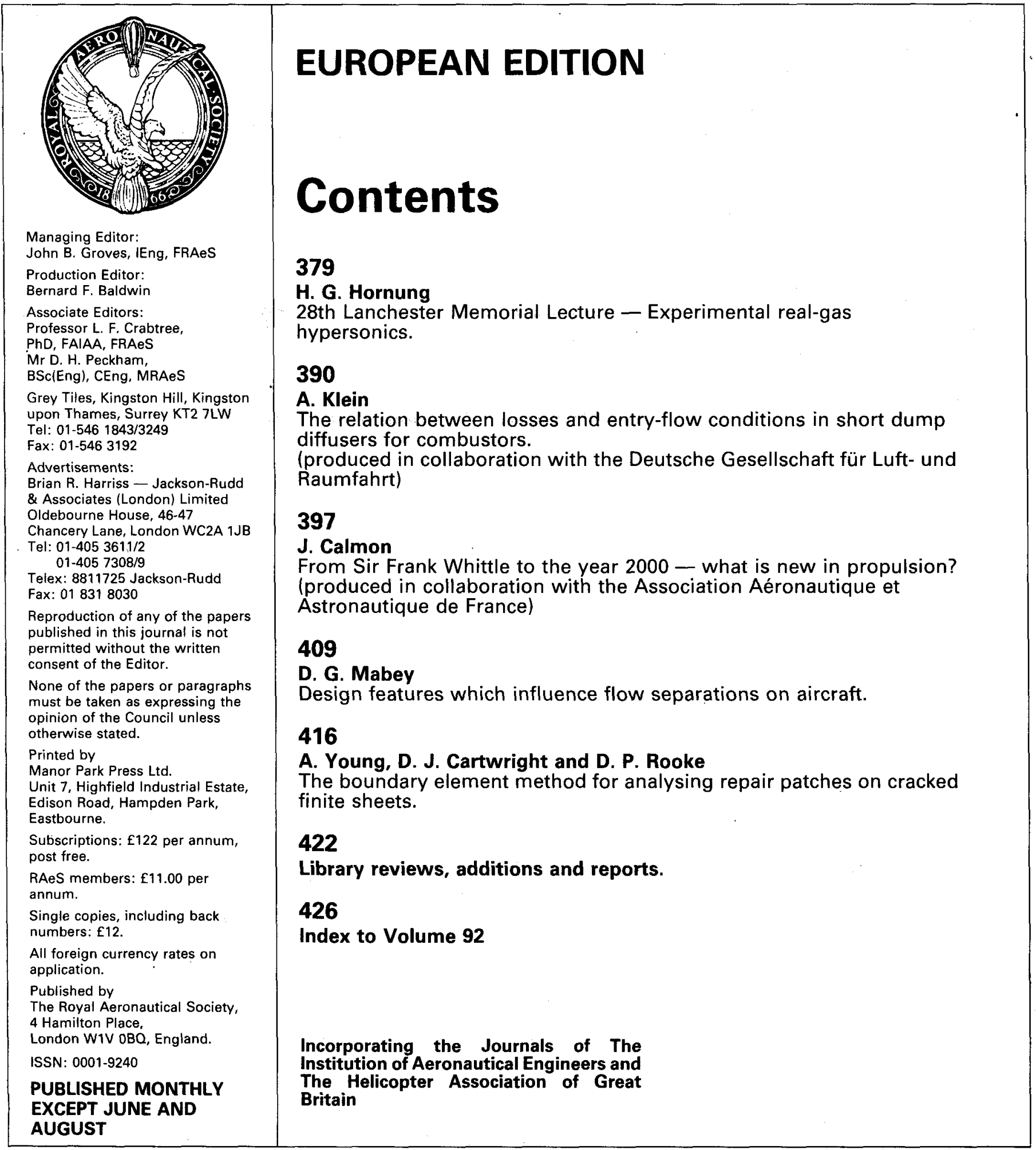




\section{THE ROYAL AERONAUTICAL SOCIETY}

Australia Branches at: Adelaide, Canberra, Melbourne and Sydney.

President: Air Vice Marshal I. T. SUTHERLAND, CEng. FRAeS. Hon. Secretary: R. D. BARKLA, PO Box 573, Mascot, NSW 2020

New Zealand Branches at: Auckland, Blenheim, Christchurch, Hamilton, Palmerston North and Wellington.

President: W. R. TANNOCK, CEng, MRAeS. Hon. Secretary: R. J. DOGGETT, PO Box 3813, Wellington.

Southern Africa President: The Hon. Mr. JUSTICE C. S. MARGO, DSO, DFC, QC, FRAeS. Hon. Secretary: N. A. H. BARRAUD, MRAeS, PO Box 630, Kelvin, Republic of South

Zimbabwe President: A. S. MATIKITI, IEng, AMRAeS. Hon. Secretary: H. W. SMITH, FRAeS, P.O. Box 1931, Harare, Zimbabwe. Tel: 4-731781.

Bedford

President: Air Vice-Marshal K. A. CAMPBELL, MSc

CEng, MRAES, RAF.

Chairman: G. I. PAULEY, CEng, MRAeS.

Hon. Secretary: I. G. HACKER, Aircraft Research

Association Ltd, Manton Lane, Bedford MK41 7PF. Tel: 023450681 .

Belfast

President: Sir PHILIP FOREMAN, CBE, FRAeS.

Hon. Secretary: W. GORDON, MRAeS, College of

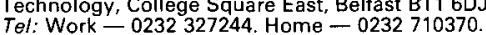

Birmingham and Wolverhampton

Président: R. G. HILTON, MA, CEng, MRAeS.

Chairman: W. G. MORRIS, IEng, AMRAeS.

Hon. Secretary: A. R. BANBURY, CEng, MIProdE, 13 Grasmere Close, Tettenhall, Wolverhampton WV6 9DP. Tel: 0902745957.

Boscombe Down

President: Air Cdre. D. L. BYWATER, FRAeS, FBIM, RAF

Chairman: R. W. NOADES, MA, MRAES

Hon. Secretary: Wg. Cdr. C. T. B. PEILE, MRAeS, RAF, RAF Handling Squadron, A\&AEE Boscombe Down, Salisbury SP4 0JF. Tel: 098023331 . Ex. 2513.

Bristol

President: D. G. ATKIN, FRAeS.
Chairman: J. E. TALBOT, FRAES

Hon. Secretary: D. C. GREENMAN, MRAeS, Aerodynamics Dept, No 7 Drawing Office, British AeroBS99 7AR. Tel: 0272 693831. Ex. 4911 .

Brough

President: M. EDWARDS, CEng, FRAeS

Chairman: P. W HOTHAM MRAES.

Hon. Secretary: J. P. NEWT́ON, CEng, MRAeS, Flight Test Department, British Aerospace, Weybridge Division, Brough, North Humberside HU15 1 EQ.
Tel: Work -0482667121 . Ex. 5821. Home -0405 83893.

\section{Cambridge}

President: Professor M. GASTER, FRS, CEng, FRAeS. Chairman: K. WESTLEY.

Hon. Secretary: T. W. CARTER, AMRAeS, Marshall of Cambridge (Engineering) Ltd, Airport Works, Cam-
bridge CB5 8RX. Tel: 022361133 . Ex. 232 or 434 .

\section{Chester}

President: S. A. E. DYKE.

President: S. A. E. DYKE,
Chairman: R. A. HUGHES, CEng, MRAeS.

Chairman: R. A. HUGHES, CEng, MRAeS.
Hon. Secretary: J. R. YOUNG, AMRAeS, British Aerospace, Civil Aircraft Division, Chester Road, Broughton, Chester CH4 ODR. Tel: 0244 535333. Ex. 161.

\section{Christchurch}

President: M. J. COBHAM, FRAeS.

Chairman: P. E. COURSE, MRAES

Hon. Secretary: R. W. SMILLIE, AMRAeS, Penny \& Giles Control Ltd, Somerford Road, Christchurch,

Coventry

President: J. B. ROBERTS, FRAeS

Chairman: Dr. B. D. HOBSON, PhD, BSc(Eng), CEng, MIMechE, MRAES

Hon. Secretary: R, I. RATCLIFF, IEng, MRAeS. 6 Erithway Rd, Green Lane, Coventry CV3 6.JT. Tel: 0203413779 . Work - 0203624000. EX. 23141.

\section{Cranwell}

President: Air Vice Marshal R. H. WOOD, OBE, RAF. Chairman: Wg. Cdr. C. T. HARROW, CEng MRAES, RAF.

Hon. Secretary: Sqn. Ldr. A. G. WILLENBRUCH MA CEng, MRAeS, MBIM, RAF, Dept. of Specialist Ground Training, RAF College, Cranwell, Sleaford, 207.

Derby

President: J. O. KEIR, FRAeS.

Hon. Secretary: C. J. ROBINSON, MRAES, c/o Compressor Technology, Rolls-Royce plc, PO Box 31 . Derby DE2 8BJ. Tel: 0332 42424. Ex. 719.

Dublin

President: R. W. O'SULLIVAN FRAeS

Chairman: Lt. Col. J. L. MOORE, MRAeS.

Hon. Secretary: J. CONNERNEY, Eng. Wing. Air Corps, Baldonnel, Co. Dublin. Tel: 592493 . Ex. 28.
Gatwick

President: A. T. PUGH, FRAeS.

Chairman: T. J. PATRICK MRAES

Hon. Secretary: A. G. SAYCE, MRAeS, Mayfield, Meath Green Lane, Horley, Surrey RH6 8JA.

\section{Glasgow}

President: Dr. A. W. BABISTER, MRAeS.

Chairman: F. J. MULLEN.

Hon. Secretary: A. REID, 42 Burntbroom Drive

Baillieston, Glasgow G69 $7 \times \mathrm{N}$

Gloucester and Cheltenham

President: M. H. SPENCE, CEng

Chairman: K. BEST, FRAeS

Hon. Secretary: N. E. LANGSTONE, CEng, MRAeS Smiths Industries Aerospace \& Defence System Ltd, Bishops Cleeve, Cheltenham, Glos GL52 4SF.
Tel: 0242673333 .

Halton

President: Gp. Capt. S. M. D. WILLIAMSON-NOBLE

Chairman: Wg. Cdr. C. J. ROWLAND, MRAeS, RAF,

Secretary: Sqn. Ldr. A. W. DEBUSE, CEng, MRAeS,

Technical Training, RAF Hatton, Aylesbury, Buck

HP22 5PG. Tel: 0296623535 . Ex. 5014.

Hatfield

President: C. B. G. MASEFIELD, FRAeS.

Chairman: L. BALTHAZOR, MRAES

Hon. Secretary: J. SAUNDERS, MRAeS, Research Dept., Civil Aircraft Division, British Aerospace, plc Hatfield, Herts. Tel: 07072 62345. Ext 3264.

\section{Heathrow}

President: LORD KING of WARTNABY, Comp. RAeS Chairman: Capt. P. J. HUNT MRAeS.

Hon. Secretary: J. D. BROWN, MRAeS, H2TBAS383 British Airways, PO Box 10, London (Heathrow) Airport, Hounslow, Middx TW6 2JA. Tel: 01-562
5958.

Isle of Wight

President: R. STANTON-JONES, FEng, MRAeS

Chairman: R. L. WHEELER, MRAES

Hon. Secretary: R. J. PERRY, Stress Office, Westland Aerospace, East Cowes. Isle of Wight. Tel: 0983 294121, Ex. 2025

\section{Loughborough}

President: Professor G. L. WILDE, OBE, FRAeS.

Chairman: S. J. RAYNER, Student RAeS

Hon. Secretary: Dr. J. B. OLLERHEAD, MRAeS, Transport Technology Dept, Loughborough Uni-
versity of Technology, Loughborough, Leics. LE11
3TU Tel: 0509223406 .

Luton

President: J. M. RAINBOW, CEng, FRAeS.

Hon. Secretary: N. G. TOOMEY, IEng, AMRAeS, c/O Monarch Aircraft Engineering Ltd (Planning Dept.) Luton International Airport, Luton, Beds. Tel: Work - 0582424211 , Ex. 226. Home - 023454185.

\section{Manchester}

President: Prof J. L. LIVESEY, CEng, MIMechE.

Chairman: J TAYLOR CEng, MRAeS.

Hon. Secretary: J. A. SHIMMIN, CEng, MRAeS 26 Denison Road, Hazel Gove, Stockport Cheshire. Tel: 061-439 5050, Ex. 3953

Medway

President: P. A. HEARNE, FRAeS

Chairman: R. T. TWINE, CEng, MRAeS.

Hon. Secretary: G. E. SIM, AMRAeS, c/o GEC Avionics Ltd, Airport Works, Rochester, Kent. Tel
063444400 .

\section{Middle Wallop}

President: Col. B. D. PORTER, CEng, MRAeS

Chairman: D. J. WILLIAMS, Eng Tech.

Secretary: C. G. TAYLOR, IEng, AMRAeS, Fairways, The Pastures, Kings Worthy, Winchester Hants SO23 7LX.

Preston

President: M. D, PARRY, FRAeS

Chairman: A. G. BARNES, FRAeS.

Hon. Secretary: I. G. JAMES, MRAeS, British Aerospace plc, Military Aircraft Div, Warton Aerodrome.
Preston, Lancs PR4 1AX. Tel: 0772 633333. Ex. 2767.
Prestwick

President: R. McINTYRE, FRAeS

Chairman: J. THOMAS, MRAES

Hon. Secretary: R. FROST, AMRAeS, 5 Burnside Road, Monkton, Ayrshire KA9 2RL.

Sherborne

President: Air Marshal Sir E. DUNN, KBE, CB, BEM CEng, FRAeS, RAF(Ret'd).

Chairman: J. L. MAYGER, IEng, AMRAeS

Hon. Secretary: C. A. FRY, FRAeS, 46 Yew Tree Close, Yeovil, Somerset BA20 2PD. Tel: Work 0935 702158. Home - 093574033 .

Southampton

President: Professor G. M. LILLEY, FRAeS

Phairman. S J NEWMAN CEng MRAES.

Hon. Secretary: D. R. J. BAXTER, CEng, MRAeS, Dept of Aeronautics and Astronautics, The University

Southend

President: Vacant

Chairman: E. B. GOLDSPINK, Assoc. RAeS.

Hon. Secretary: D. M. GLADSTONE, MRAeS, 36

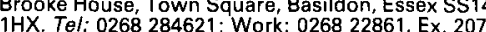

\section{Stevenage}

President: J. PARKHOUSE, CBE, FRAeS

Chairman: N. MUIR, CEng, FRAeS.

Hon. Secretary: A. CRESSWELL, BAe Dynamics Divi sion, P.O. Box 19, Six Hills Way, Stevenage, Herts. Tel: 0438753709 .

Swindon

President: W. GILLESPIE, CEng, MRAES

Chairman: Sqn. Ldr. J. J. D. RICHARDSON, CEng, MRAeS.

Hon. Secretary: V. SIMON, 20 Hawkswood, Coving ham Park, Swindon, Wilts. Tel: 079325350.

\section{Weybridge}

President: N. V. BARBER, BA, MSc, FRAeS.

Chairman: B. W PAYNE, CEng, FRAeS.

Hon. Secretary: K. J. DAVIES, CEng, MRAeS and $\checkmark$. MITCHELL, Corporate Headquarters, British Aerospace plc, Brooklands Road, Weybridge, Surrey KT13 OSF. Tel: 093245522 .

Yeovil

President: V. A. B. ROGERS, CBE, FRAES

Chairman: R. I. CASE, CEng, FRAeS

Hon. Secretary: F. N. R. BALLAM, CEng, MRAeS, Box 269, Westland Helicopters Ltd, Yeovit, Somerset
BA20 2YB. Tel: 0935703779 .

\section{OVERSEAS BRANCHES}

Cyprus

Chairman: Air Vice-Marshal G. A. WHITE.

Secretary: C. PERICLEOUS, AMRAES, 8 Georgiou Christodolou, Acropolis, Nicosia, Cyprus.

\section{Hong Kong}

Hon. Secretary: M. J. POMFRET, MRAeS, MME Dept, HK Polytechnic, Kowloon, Hong Kong. Tel: Office: 3-638344. Ex. 765. Home: 3-7213732.

Malaysia

Chairman: Brig. Gen. Dato' M. MUSLIM AYOB RMAF, CEng, MRAeS

Hon. Secretary: Lt. Col. CHEE ENG BOON, RMAF CEng, MRAeS, Dept of the Air Force, Ministry of Defence, Jalan Padang Tembek, 50634 Kuala Lum pur, Malaysia. Tel: 03-2354010.

\section{Pakistan}

Chairman: S. AJAZ ALI, CEng, FRAeS.

Hon. Secretary: Dr. S. A. HASAN, CEng, MRAeS, PAF PIA Training Centre, Karachi Airport-75200, Pakistan. 